\title{
O combate a hipertensão arterial na estratégia e saúde da família: uma revisão bibliográfica
}

\author{
Combating arterial hypertension in family strategy and health: a bibliographic review \\ Combatir la hipertensión arterial en la estrategia familiar y la salud: una revisión \\ bibliográfica
}

Josimá Lima Oliveira $^{1 *}$, Lusiane Lima Oliveira ${ }^{1}$, Thiago Coelho Scherrer de Souza1 ${ }^{1}$ Sônia Maria da Costa Barreto'1.

\section{RESUMO}

Objetivo: Abordar as políticas de combate e tratamento da hipertensão arterial na Estratégia e Saúde da Família (ESF). Revisão bibliográfica: A hipertensão ou pressão alta é uma doença crônica caracterizada por pressão alta nas artérias. Isso acontece quando os valores de pressão máxima e mínima são iguais ou superiores a 140/90 mmHg. Os pontos chamados ESF, são locais que compõem a atenção primária à saúde, e que prestam os primeiros atendimentos e diligenciam os procedimentos necessários, é o primeiro contato do paciente com a saúde pública. Também é importante ressaltar que é nas ESFs que os tratamentos de controle e as políticas de combate à inúmeras doenças acontecem, e no caso, a hipertensão arterial é uma delas. Considerações finais: As equipes de profissionais, as casas de saúde pública e todos os investimentos do Estado no combate à HAS, são uma forma de buscar o aumento da qualidade de vida e combater mortes precoces. Com base na análise inicial, objetiva-se nesta revisão apontar a importância da Estratégia e Saúde da Família no tratamento e na prevenção da Hipertensão Arterial.

Palavras-chave: Hipertensão arterial, HAS, Estratégia e saúde da família.

\begin{abstract}
Objective: To address policies to combat and treat arterial hypertension in the Family Health and Strategy (FHS). Bibliographic review: Hypertension or high blood pressure is a chronic disease characterized by high blood pressure. This happens when the maximum and minimum pressure values are equal to or greater than 140/90 $\mathrm{mmHg}$. The points called ESF, are places that comprise primary health care, and that provide the first care and perform the necessary procedures, is the patient's first contact with public health. It is also important to note that it is in the FHS that control treatments and policies to combat numerous diseases occur, and in this case, arterial hypertension is one of them. Final considerations: The teams of professionals, the public health houses and all the investments of the State in the fight against $\mathrm{SAH}$, are a way of seeking to increase the quality of life and fight early deaths. Based on the initial analysis, the objective of this review is to point out the importance of Family Health and Strategy in the treatment and prevention of Arterial Hypertension.
\end{abstract}

Key words: Arterial hypertension, SAH, Strategy and family health.

\section{RESUMEN}

Objetivo: Abordar políticas de combate y tratamiento de la hipertensión arterial en la Estrategia y Salud de la Familia (ESF). Revisión bibliográfica: La hipertensión o presión arterial alta es una enfermedad crónica caracterizada por presión arterial alta. Esto sucede cuando los valores de presión máxima y mínima son iguales o superiores a 140/90 $\mathrm{mmHg}$. Los puntos denominados ESF, son lugares que comprenden la atención primaria de salud, y que brindan la primera atención y realizan los trámites necesarios, es el primer contacto del paciente con la salud pública. También es importante señalar que es en la ESF donde se producen los tratamientos y políticas de control para combatir numerosas enfermedades, y en este caso la hipertensión arterial es una de ellas. Consideraciones finales: Los equipos de profesionales, las casas de salud pública y todas las inversiones del Estado en la lucha contra la HAS, son una forma de buscar aumentar la calidad de vida y combatir las muertes tempranas. A partir del análisis inicial, el objetivo de esta revisión es señalar la importancia de la Salud y la Estrategia de la Familia en el tratamiento y prevención de la Hipertensión Arterial.

Palabras clave: Hipertensión arterial, HSA, Estrategia y salud familiar.

${ }^{1}$ Faculdade Vale do Cricaré (FVC), São Mateus - ES. * E-mail: josima@bol.com.br 


\section{INTRODUÇÃO}

Atualmente, as doenças crônico-degenerativas são consideradas a principal causa de morbimortalidade em países desenvolvidos e em desenvolvimento. Muitos fatores de risco estão relacionados ao desenvolvimento desse tipo de doença, como tabagismo, falta de exercícios, alimentação insuficiente, etc. Nesse caso, a hipertensão arterial é uma doença clínica multifatorial caracterizada por níveis persistentemente elevados de pressão, que causa alterações na função e/ou estrutura dos órgãos-alvo (coração, cérebro, rins e vasos sanguíneos) e alterações no metabolismo. Razões comuns para o aumento do risco de eventos cardiovasculares fatais e não fatais (SILVA MGC, et al., 2018).

A hipertensão arterial é um aumento anormal da pressão produzida pelo sangue que circula nas artérias do corpo - a longo prazo, a doença também é chamada de pressão alta. Para atingir todas as partes do corpo, o sangue bombeado do coração exerce forças naturais nas paredes internas das artérias. Os vasos sanguíneos, por sua vez, oferecem alguma resistência a essa passagem. É essa a atividade que determina a pressão arterial. A pressão varia ao longo do dia, em uma pessoa repousada, torna-se mais baixa, à medida que nos movimentamos, os valores aumentam porque o cérebro alerta que o corpo precisa de mais energia (VEJA, 2019).

A hipertensão arterial sistêmica (HAS) continua sendo um dos maiores desafios de saúde pública em todo o mundo. Um estudo populacional estimou que $25,6 \%$ da população dos EUA tem hipertensão (medida por medidas de pressão alta ou relacionada ao uso de medicamentos anti-hipertensivos), chegando a $29 \%$ entre os mais pobres e $39 \%$ entre as mulheres negras. No Brasil, um estudo de revisão estimou a incidência de HAS em $25 \%$ na população acima de 20 anos. A atenção programada parece desempenhar um papel importante na melhoria do controle da pressão arterial. Estudo realizado em serviços de atenção básica mostrou que os jovens que participavam de consultas regulares apresentavam níveis pressóricos mais baixos (HELENA ETS, et al., 2010).

A Estratégia Saúde da Família (ESF), que está em vigor no Brasil desde 1994, gerou um modelo descentralizado de atenção preventiva e corretiva no país, com foco nas condições de saúde do bairro. Essas ações possibilitam que todo cidadão brasileiro tenha acesso a serviços médicos, ferramentas de atendimento que auxiliem na efetivação das ações e promovam a segurança dos usuários por meio de serviços prestados por profissionais de saúde. Porém, o panorama atual da assistência no âmbito da Atenção Primária à Saúde (APS) vem se modificando ao longo do trabalho, exigindo diversidade na gestão nas ações que visam potencializar a assistência prestada. No monitoramento da população de doenças crônicas não transmissíveis (DCNT), devido ao elevado número de óbitos em 2012, estimado em mais de 38 milhões de pessoas, a APS desempenha um papel vital no sucesso do controle e tratamento dessas doenças (RÊGO AS, et al., 2018).

Além de ser uma doença, a HAS também é o fator de risco mais comum e reversível para doenças cardiovasculares. Sua ocorrência é uma grande causa de morte prematura e redução da qualidade de vida com alto grau de limitação e incapacidade - levando a uma grande demanda por cuidados de saúde, absenteísmo no trabalho, aumento de custos para famílias, comunidades e sistemas de saúde e previdência social. Os fatores de risco incluem genética, raça, idade, sexo, excesso de peso, estresse, estilo de vida sedentário, ingestão elevada de sódio, baixo nível de escolaridade, existência de comorbidades relacionadas, características de histórico e residência. A doença é assintomática e isso pode atrasar o seu diagnóstico (OLIVEIRA BLCA, et al., 2020).

O tratamento adequado requer avaliação clínica adequada e regular, o que não é comum entre pessoas com níveis de renda mais baixos, pessoas com baixa escolaridade ou residentes em áreas remotas e pessoas com infraestrutura social e de saúde precária. Por outro lado, muitos medicamentos, seu alto custo, efeitos colaterais e tempo insuficiente, para orientação do paciente levam à não insistência no tratamento. Poucas consultas de saúde, não adesão ao tratamento, medicação incorreta e poucas mudanças no estilo de vida e nos comportamentos ligados à saúde dos hipertensos também são considerados os principais fatores de controle ineficaz da HAS. Esses fatores ainda aumentam o risco de complicações causadas pela doença, o que pode levar a maiores taxas de internação (OLIVEIRA BLCA, et al., 2020). 
Observando os principais fatores causadores da hipertensão arterial, além dos fatores potencializadores da sua existência, pode-se averiguar quais importantes políticas sociais colaboram para o controle e a prevenção. As equipes de profissionais, as casas de saúde pública e todos os investimentos do Estado no combate à HAS, são uma forma de buscar o aumento da qualidade de vida e combater mortes precoces. Com base na análise inicial, objetiva-se nesta revisão apontar a importância da Estratégia e Saúde da Família no tratamento e na prevenção da Hipertensão Arterial.

\section{REVISÃO BIBLIOGRÁFICA}

O modelo de atenção à saúde no Brasil é derivado de um processo de descentralização e regionalização, que, entre outras medidas, implementa um plano de saúde da família (PSF). A atuação de equipes multiprofissionais em áreas designadas de assistência, prevenção e promoção da saúde tem facilitado o acesso das pessoas aos serviços. Dentre os programas atendidos pelo PSF, o Hiperdia se destaca por atender os usuários acometidos pelas duas doenças crônicas não transmissíveis (DCNT) mais prevalentes, a hipertensão e o diabetes, que, devido ao seu caráter crônico, afetam a qualidade e o estilo de vida desses usuários (RIBEIRO IJS, et al., 2015).

A HAS não é apenas uma doença, mas também um fator de risco e agravante para outras doenças. Além disso, não tem cura e requer tratamento vitalício. Percebe-se que, desde que aplicado de maneira correta como um todo, o conhecimento existente sobre a HAS e medidas de controle é um elemento que pode reduzir muito as mortes por essa doença. A prática de controle dessa doença, silenciosa e progressiva, pode ser um tratamento por meio do uso de certos medicamentos prescritos pelo médico; e / ou não tratada, onde requer mudança no estilo de vida da pessoa e adoção de comportamentos mais saudáveis. Se não for tratada, a hipertensão pode ter consequências graves para alguns órgãos vitais. Portanto, é um dos mais graves problemas de saúde pública. Estima-se que $40 \%$ dos acidentes vasculares cerebrais e $25 \%$ dos ataques cardíacos em pacientes com hipertensão poderiam ser evitados (SILVA MLB e BOUSFIELD ABS, 2012).

Sabemos que a obesidade e o ganho de peso são fatores de risco fortes e independentes para hipertensão, por isso estima-se que $60 \%$ das pessoas com hipertensão têm mais de $20 \%$ de excesso de peso. Há um aumento da prevalência de hipertensão na população em relação à ingestão de $\mathrm{NaCl}$ e uma baixa ingestão alimentar de cálcio e potássio, o que pode contribuir para o risco de hipertensão. Quando se trata de fatores ambientais, como consumo de álcool, estresse psicoemocional e baixos níveis de atividade física, eles também podem contribuir para a hipertensão. A hipertensão também é chamada de assassino silencioso, porque muitos pacientes não apresentam sintomas da doença, tornando o diagnóstico difícil, e o diagnóstico geralmente se deve a complicações (MAGRINI DW e MARTINI JG, 2012).

Com o advento da Portaria no 648, de 28 de março de 2006 (revogada pela portaria 2.488, de 21 de outubro de 2011), que ratifica a Política Nacional de Atenção Básica e constitui o regimento que rege sua organização, inclusive o Programa Saúde da Família (PSF), garante que a equipe multiprofissional seja, no máximo, responsável por, 4.000 habitantes, com média de 3.000 habitantes indicados, com jornada de trabalho de 40 horas semanais para todos os associados e composta por, no mínimo, médico, enfermeiro ou técnico de enfermagem e agente comunitário de saúde.

Neste contexto, o médico desempenha um papel cada vez mais decisivo e proativo na definição das responsabilidades de cuidar da população, bem como na promoção e proteção da saúde humana nas suas várias dimensões. Desta forma, o atendimento médico se torna um componente básico do sistema de saúde local que exibe a sua reflexão a nível regional e nacional, procurando assim intensificar debates e novos sentidos (CANALES DL, 2014).

A referida portaria dispõe que: Art. 1ํ Aprovar a Política Nacional de Atenção Básica, com vistas à revisão da regulamentação de implantação e operacionalização vigentes, nos termos constantes dos Anexos a esta Portaria. 
São necessárias à realização das ações de Atenção Básica nos municípios e Distrito Federal: I - Unidades Básicas de Saúde (UBS) construídas de acordo com as normas sanitárias e tendo como referência o manual de infra estrutura do Departamento de Atenção Básica/SAS/ MS; II - as Unidades Básicas de Saúde: a) devem estar cadastradas no sistema de Cadastro Nacional vigente de acordo com as normas vigentes; b) Recomenda-se que disponibilizem, conforme orientações e especificações do manual de infra estrutura do Departamento de Atenção Básica/SAS/ MS: 1. consultório médico/enfermagem, consultório odontológico e consultório com sanitário, sala multiprofissional de acolhimento à demanda espontânea, sala de administração e gerência e sala de atividades coletivas para os profissionais da Atenção Básica; 2. área de recepção, local para arquivos e registros, sala de procedimentos, sala de vacinas, área de dispensação de medicamentos e sala de armazenagem de medicamentos (quando há dispensação na UBS), sala de inalação coletiva, sala de procedimentos, sala de coleta, sala de curativos, sala de observação, entre outros (BRASIL, 2011).

\section{O combate a hipertensão arterial através de políticas de saúde}

Estudos epidemiológicos demonstraram aumento na incidência de hipertensão sistêmica (HAS) na faixa etária pediátrica, enquanto outros estudos demonstraram aumento na incidência de $1 \%$ a $11 \%$ nessa população. É importante reconhecer essa realidade para atuar precocemente na prevenção de eventos cardiovasculares, pois a alteração da pressão arterial na infância pode predizer HAS na vida adulta. Entre os vários fatores que contribuem para a ocorrência de HAS estão os fatores de risco constitucionais: idade, sexo, raça/cor e história familiar, bem como fatores de risco ambientais para um estilo de vida sedentário, sobrepeso/obesidade, ingestão de alimentos não saudáveis (excesso de sal, gordura animal, preferência por carboidratos simples e complexos, consumo diário de mais de $100 \mathrm{ml}$ de café ou bebidas com cafeína, abuso de álcool, estresse descontrolado e tabagismo (SANTOS ZMSA, et al., 2011).

Dentre os fatores de risco relacionados mais importantes, alguns são considerados imutáveis, como idade, sexo, raça, fatores socioeconômicos e fatores genéticos. Na população passível de mudança, o estilo de vida insuficiente está relacionado à maior prevalência de hipertensão e menor proteção contra doenças. Portanto, os elementos básicos de prevenção e tratamento da HAS são mudanças no estilo de vida, como adoção de dieta hipocalórica, perda de peso, prática de exercícios físicos e redução da ingestão de álcool e/ou sal. Este é o método mais eficaz e eficaz e mais barato na saúde pública. $O$ efeito de uma dieta saudável (rica em frutas e vegetais; pobre em gordura) sobre os níveis de pressão arterial é bem conhecido. Os fatores nutricionais associados à alta prevalência de HAS incluem ingestão excessiva de álcool e sódio e sobrepeso. Recentemente, o consumo de potássio, cálcio e magnésio também foi relacionado, o que reduzirá gradualmente os níveis de pressão arterial à medida que envelhecemos (OLIVEIRA EP, et al., 2012).

Fumar é considerado um fator de risco para hipertensão em adultos. Estudos anteriores são conclusivos na confirmação dos efeitos deletérios do tabagismo na Variabilidade da Frequência Cardíaca (VFC) de adultos, o que indica que a redução da VFC é um dos muitos mecanismos que levam os fumantes a desenvolver hipertensão arterial. De fato, em adultos, a redução da VFC parece preceder o aparecimento desta doença. No entanto, nos primeiros estágios da vida, a relação entre tabagismo e doenças cardiovasculares ainda é pouco compreendida. Estudos demográficos não encontraram associação entre tabagismo em adolescentes e hipertensão. No entanto, esse comportamento de risco pode ter um impacto negativo na VFC, o que pode ser um gatilho para hipertensão na idade adulta (GONDIM RM, et al., 2015).

O envelhecimento, antes visto como um fenômeno, passou a fazer parte da maior parte da realidade social, e esse crescimento é mais pronunciado nas sociedades ocidentais. É caracterizada por um processo multifatorial que é afetado por aspectos psicológicos, sociais, biológicos e funcionais, o que reduz a capacidade física e prejudica o desempenho na vida diária, junto com essa rápida transição demográfica, as doenças crônicas não transmissíveis (DCNT) aumentaram. A hipertensão arterial sistêmica é a doença 
crônica mais comum no Brasil e no mundo. É considerada o principal fator de risco para doenças cardiovasculares. Em 2011, a doença causou 33\% dos óbitos no Brasil, afetando homens e mulheres de diversas idades, sendo que os idosos apresentam maior prevalência (CASSIANO AN, et al., 2020).

O exercício é uma atividade física planejada, estruturada e repetitiva com o objetivo final ou indireto de melhorar ou manter a saúde e a forma física, e que pode fornecer benefícios agudos e crônicos. Entre eles, está a melhora da condição física; redução da perda de massa óssea e muscular; aumento da força, coordenação e equilíbrio; redução da incapacidade funcional, intensificação de pensamentos negativos e doenças físicas; e promover melhoria do bem-estar e do humor, além de diminuir a pressão arterial (PA) pós-exercício em relação aos níveis pré-exercício. O efeito protetor do esforço físico vai além da redução da pressão arterial, está associado à redução dos fatores de risco cardiovascular, e menor morbimortalidade em relação a pessoas fisicamente ativas e pessoas com menor aptidão física, o que explica a recomendação para prevenção e tratamento da hipertensão primária (NOGUEIRA IC, et al., 2012).

Estudos epidemiológicos transversais ou longitudinais correlacionam o consumo crônico de etanol com o desenvolvimento de hipertensão arterial independentemente do tipo de bebida alcoólica, porém, dependendo dos padrões de consumo e não relacionados a fatores como idade, sexo, raça e obesidade. Vários padrões de consumo de álcool existem em diferentes culturas e entre as diferentes populações no que diz respeito aos hábitos de consumo que vão desde o consumo leve a moderado durante ou fora das refeições e ao consumo pesado, que é uma situação muito preponderante em alguns países europeus. Há riscos e benefícios no consumo de álcool e correlação com a mortalidade em pessoas com hipertensão. $O$ consumo de álcool leve a moderado parece ter um efeito cardioprotetor na redução da mortalidade cardiovascular em pessoas com hipertensão e com níveis de pressão de hipertensão em comparação com abstêmios (SOUZA DSM, 2014).

\section{O controle da hipertensão arterial na ESF}

Estudos avaliados mostram que os indicadores de controle da hipertensão em nosso país eram muito diferentes. O controle da hipertensão é resultado de um sistema complexo que envolve aspectos biológicos, socioeconômicos, culturais e de saúde. A diversidade da população brasileira torna necessária a ampliação do conhecimento sobre o controle da hipertensão e seus determinantes nas diferentes regiões do Brasil. Não se pode negar que o controle da hipertensão geralmente não é satisfatório. A provável explicação está relacionada à redução insuficiente dos níveis pressóricos devido a uma proposta de tratamento ineficaz. Outra possibilidade de controle insatisfatório da pressão arterial é com pouca ou nenhuma adesão ao tratamento (PINHO NA e PIERIN AMG, 2013).

Alguns estudos mostram que os países com grande população rural apresentam um nível de conhecimento, tratamento e controle inaceitavelmente baixo. Em um desses estudos, realizado em dois países rurais da África, entre 2.678 participantes de um estudo na Nigéria, a incidência de HAS foi de $19,3 \%$, sendo que $92 \%$ não sabiam que se tratava de uma doença hipertensiva, $2 \%$ estavam em tratamento e apenas 3\% com PA controlada. Taxas alarmantes também foram encontradas no Quênia entre 2.111 participantes, cuja incidência de HAS foi de 21,4\%; $83 \%$ desconheciam seu estado de hipertensão; $9 \%$ estavam em tratamento; e apenas 2,6\% tinham PA sob controle (MAGNABOSCO P, et al., 2017).

O controle da pressão arterial em pacientes hipertensos está intimamente relacionado à adesão ao tratamento prescrito. A não adesão à medicação é uma preocupação importante para profissionais de saúde e gestores, e pesquisas são necessárias para ajudar a melhorar a adesão aos medicamentos antihipertensivos, especialmente em pacientes hipertensos com alto risco cardiovascular. Nesses pacientes, 0 controle da doença pode reduzir significativamente a mortalidade dessas doenças e o custo do tratamento de complicações. Além disso, o controle inadequado da pressão arterial pode aumentar o risco de uma crise de hipertensão que requeira hospitalização. Vários fatores relacionados à adesão incluem as características do paciente, a qualidade da relação médico-paciente, a gravidade da doença, o acesso a cuidados médicos e fatores específicos relacionados às prescrições de medicamentos (SOUZA CS, et al., 2014).

A grande lacuna entre as estimativas epidemiológicas e os dados sobre os serviços de registro de pacientes com hipertensão e diabetes indica que as medidas de controle desses problemas não são 
eficazes, principalmente na área de atenção primária e também indicam a necessidade de gestão e atenção à saúde. As atividades realizadas por profissionais precisam ser monitoradas de forma mais rigorosa. $O$ HiperDia é um sistema de cadastro e monitoramento de hipertensos e diabéticos, no qual os profissionais médicos são responsáveis pelo atendimento aos usuários e pelo preenchimento dos dados. Sua finalidade é monitorar os pacientes captados no Plano Nacional de Redirecionamento de HAS e DM e gerar informações periódicas e contínuas para a obtenção, cadastramento e distribuição de medicamentos (FILHA FSSC, et al., 2014).

A educação em saúde é uma ferramenta extremamente importante para promover a saúde humana e a saúde pública. No início do século 20 , o foco da atenção do governo em epidemias como varíola, malária, febre amarela e depois, peste fez com que o mesmo investisse na importância de se instruir sobre higiene pessoal e considerar mudanças de comportamento diante da sociedade. Ao incluir as diretrizes, a importância da saúde pública na educação ganhou um novo modelo. Foi promovido com a emissão da "Carta de Ottawa", que afirmava que saúde é oferecer opções para a população para que ela possa melhorar sua forma e expectativa de vida (NICOLAU S, et al., 2018)

Percebe-se que o campo de desenvolvimento da educação e da prática de enfermagem ao portador de HAS e diabetes Mellitus (DM) está inserido na "Estratégia Saúde da Família-ESF", que se define como uma série de condutas da APS voltadas para a prevenção dessas doenças. O desafio de controlar e prevenir a HAS e o DM é colocado pela equipe médica de base, onde o enfermeiro exercerá suas funções profissionais e trabalhará com a equipe médica, o que faz com que 0 trabalho junto à comunidade desenvolva a diversidade socioeconômica (NICOLAU S, et al., 2018).

A finalidade da informação em saúde inclui a identificação de problemas de saúde individuais e coletivos, que fornecem ferramentas que podem armazenar, gerar, organizar e analisar os dados necessários para definir problemas e riscos de saúde, bem como subsídios para novas práticas de saúde e gestão. É imprescindível analisar e investigar constantemente as informações sobre questões de saúde, esses sistemas de informação devem atender às necessidades e antecipar as necessidades dos usuários, coletar, organizar, distribuir e disponibilizar informações para uso em procedimentos médicos. Na atenção básica, de acordo com as diretrizes de planejamento, foi implantado em janeiro de 2002 o Sistema de Informação em Saúde de Hi-perdia (SIS/Hiperdia), que cadastra e monitora usuários com hipertensão arterial (HA) e diabetes (DM). O foco em AH e DM é reorganizado. O objetivo é cadastrar os usuários de hipertensão e diabetes e fornecer regularmente os medicamentos necessários, além de monitorar e avaliar a morbimortalidade dessas doenças (VALLE WAC, et al., 2011).

\section{CONSIDERAÇÕES FINAIS}

A literatura é vasta e aponta claramente a importância de manter e ampliar as políticas públicas de saúde próximas da população. As formas de se alcançar este objetivo vão muito além de fornecer as condições mínimas para o tratamento, mas também em divulgar e instruir a população para que ela possa buscar melhores condições. A hipertensão arterial é uma das doenças que mais causa morte no Brasil e no mundo, não somente pelos seus efeitos, mas muito se deve ao agravamento em outras situações de fragilidade. A Estratégia e Saúde da Família é uma importante referência de tratamento, e leva aos brasileiros, muito pela proximidade com as comunidades, as condições mínimas para os cuidados com a saúde cardíaca.

\section{REFERÊNCIAS}

1. BRASIL. 2011. In: PORTARIA № 2.488, DE 21 DE OUTUBRO DE 2011: Aprova a Política Nacional de Atenção Básica, estabelecendo a revisão de diretrizes e normas para a organização da Atenção Básica, para a Estratégia Saúde da Família (ESF) e o Programa de Agentes Comunitários de Saúde (PACS). Disponível em: http://bvsms.saude.gov.br/bvs/saudelegis/gm/2011/prt2488_21_10_2011.html. Acesso em: 16 de set. 2020.

2. CANALES DL. Estratégias de saúde para o controle da hipertensão arterial sistêmica nos pacientes da unidade básica de saúde palmeiras, Central-Bahia. Projeto de Intervenção (Especialização em Saúde da Família - Programa Mais Médicos para o Brasil/PROVAB). Universidade Federal do Mato Grosso do Sul, Central-Bahia, $2014 ; 34$ p. 
3. CASSIANO AN, et al. Efeitos do exercício físico sobre o risco cardiovascular e qualidade de vida em idosos hipertensos. Ciência \& Saúde Coletiva, 2020; 25(6): 2203-2212.

4. FILHA FSSC, et al. Avaliação do controle de hipertensão e diabetes na Atenção Básica: perspectiva de profissionais e usuários. Saúde Debate, 2014; 38(1): 265-278.

5. GONDIM RM, et al. O tabagismo e o fumo passivo estão relacionados com a variabilidade da frequência cardíaca em adolescentes homens?. Einstein, 2015; 13(1): 27-33.

6. HELENA ETS, et al. Avaliação da assistência a pessoas com hipertensão arterial em Unidades de Estratégia Saúde da Família. Saúde e Sociedade, 2010; 19(3): 614-626.

7. MAGNABOSCO P, et al. Prevalência e controle da hipertensão arterial: estudo comparativo entre população urbana e rural. Revista Mineira de Enfermagem, 2017; 21(1): 1-11.

8. MAGRINI DW, MARTINI JG. Hipertensão arterial: principais fatores de risco modificáveis na estratégia saúde da família. Enfermaría Global, 2012; 26(1): 354-363.

9. NICOLAU S, et al. Práticas de educação em saúde realizadas por enfermeiros para pacientes do programa hiperdia. J Manag Prim Health Care, 2018; 9(9): 1-19.

10. NOGUEIRA IC, et al. Efeitos do exercício físico no controle da hipertensão arterial em idosos: uma revisão sistemática. Revista Brasileira de Geriatria e Gerontologia, 2012; 15(3): 587-601.

11. OLIVEIRA BLCA, et al. A influência da Estratégia Saúde da Família no uso de serviços de saúde por adultos hipertensos no Brasil. Revista Brasileira de Epidemiologia, 2020; 23(1): 1-14.

12. OLIVEIRA EP, et al. A variedade da dieta é fator protetor para a pressão arterial sistólica elevada. Arquivos Brasileiros de Cardiologia, 2012; 98(4): 338-343.

13. PINHO NA, PIERIN AMG. O controle da hipertensão arterial em publicações brasileiras. Arquivos Brasileiros de Cardiologia, 2013; 101(3): 65-73.

14. RÊGO AS, et al. Adesão/vínculo de pessoas com hipertensão arterial na Estratégia Saúde da Família. Rev Bras Enferm [Internet], 2018; 71(3): 1093-1100.

15. RIBEIRO IJS, et al. Qualidade de Vida de hipertensos atendidos na Atenção Primária à Saúde. Saúde e Debate, 2015; 39(105): 432-440.

16. SANTOS ZMSA, et al. Atuação dos pais na prevenção da hipertensão arterial: uma tecnologia educativa em saúde. Ciência \& Saúde Coletiva, 2011; 16(11): 4385-4394.

17. SILVA MGC, et al. Hipertensão arterial e cuidados com a saúde: concepções de homens e mulheres. Psicologia, Saúde \& Doenças, 2018; 19(2): 435-452.

18. SILVA MLB, BOUSFIELD ABS. Representações sociais da hipertensão arterial. Temas em Psicologia, 2012; 24(3): 895-909.

19. SOUZA CS, et al. Controle da Pressão Arterial em Hipertensos do Programa Hiperdia: Estudo de Base Territorial. Arquivos Brasileiros de Cardiologia, 2014; 102(6): 571-578.

20. SOUZA DSM. Álcool e hipertensão. Aspectos epidemiológicos, fisiopatológicos e clínicos. Rev Bras Hiperten, 2014; 21(2): 83-86.

21. VALLE WAC, et al. Hiperdia, Sistema de Informação em Saúde, produção de conhecimento para melhoria da prática de enfermagem na Atenção Básica. Enfermagem Brasil, 2011; 10(2): 119-125.

22. VEJA. 2019. In: HIPERTENSÃO: causas, sintomas, diagnóstico e como baixar a pressão, Veja Saúde; São Paulo. Disponível em: https://saude.abril.com.br/medicina/hipertensao-causas-sintomas-diagnostico-e-como-baixar-apressao/. Acesso em: 11 de ago. 2020. 